Faculté des sciences sociales École de psychologie

SEPTEMBRE 2018 - VOL. $8 \mathrm{~N}^{\circ} 2$

\title{
PROFILS DE RÉPERCUSSIONS SEXUELLES CHEZ DES FEMMES VICTIMES D'AGRESSION SEXUELLE À L'ÂGE ADULTE
}

Laurie CÔTÉ1,*, Stéphanie DUCHESNE¹, Justine MITCHELL',

Marie-Pier VAILLANCOURT-MOREL' ${ }^{1}$ Stéphane SABOURIN ${ }^{1}$ \& Elodie HAMEL ${ }^{1}$

1 École de psychologie, Université Laval, Québec, Canada

*laurie.cote.6@ulaval.ca

\section{Pour citer l'article}

Côté, L., Duchesne, S., Mitchell, J., Vaillancourt-Morel, M.-P., Sabourin, S., \& Hamel, E. (2018). Profils de répercussions sexuelles chez des femmes victimes d'agression sexuelle à l'âge adulte. Psycause : Revue scientifique étudiante de l'École de psychologie de l'Université Laval, 8(2), 29-32. 
Prinz, R. J. (2016). Parenting and family support within a broad child abuse prevention strategy: Child maltreatment prevention can benefit from public health strategies. Child Abuse \& Neglect, 51, 400-406. doi:10.1016/j. chiabu.2015.10.015

Prinz, R. J., Sanders, M. R., Shapiro, C. J., Whitaker, D. J., \& Lutzker, J. R. (2016). Addendum to «Population-based prevention of child maltreatment: The U.S. Triple P system population trial». Prevention Science, 17, 410-416. doi:10.1007/s11121-016-0631-x
Shapiro, C. J., Prinz, R. J., \& Sanders, M. R. (2010). Population-based provider engagement in delivery of evidence-based parenting interventions: challenges and solutions. Journal of Primary Prevention, 31, 223-234. doi:10.1007/s10935-010-0210-z

Shelton, K.K., Frick, P. J., \& Wootton, J.M. (1996). Assessment of parenting practices in families of elementary school-age children. Journal of Clinical Child Psychology, 25, 317-329. doi:10.1207/s15374424jccp2503_8

Waldfogel, J., Craigie, T. A., \& Brooks-Gunn, J. (2010). Fragile families and child wellbeing. Future Child, 20, 87-112. doi:10.1353/ foc. 2010.0002

\section{PROFILS DE RÉPERCUSSIONS SEXUELLES CHEZ DES FEMMES VICTIMES D'AGRESSION SEXUELLE À L'ÂGE ADULTE}

Laurie CÔTÉ, Stéphanie DUCHESNE, Justine MITCHELL, Marie-Pier VAILLANCOURT-MOREL, Stéphane SABOURIN \& Elodie HAMEL École de psychologie, Université Laval, Québec, Canada

\section{Introduction}

L'agression sexuelle à l'âge adulte (ASA) est un acte de nature sexuelle avec ou sans contact direct posé par un individu sur une victime âgée d'au moins 16 ans sans son consentement (Gouvernement du Québec, 2001). De nombreuses séquelles sexuelles sont observées chez les femmes à la suite d'une ASA. En effet, ces femmes rapportent des taux plus élevés de détresse sexuelle (Conoscenti \& McNally, 2006), de compulsion sexuelle (Brown et coll., 2003), d'évitement sexuel (Gidycz \& Kelley, 2016) et de perturbations sexuelles (O'Driscoll \& Flanagan, 2016). Ces femmes vivent également une baisse de satisfaction sexuelle (Offman \& Matheson, 2004) et de la fonction sexuelle (Turchik \& Hassija, 2014). Certaines variables sont reconnues pour avoir une influence sur les répercussions sexuelles à la suite d'une ASA. Comparativement aux femmes célibataires, les femmes mariées sont moins susceptibles d'adopter des comportements sexuels à risque (Mittal et coll., 2013). De plus, les femmes de bas niveau socioéconomique s'engagent davantage dans des relations sexuelles non protégées (Wingood \& DiClemente, 1998). Aussi, Burri, Schweitzer et O'Brien (2014) rapportent que, dans la population générale, de hauts niveaux d'anxiété d'abandon et d'évitement de l'intimité affectent négativement l'orgasme, la satisfaction sexuelle etlefonctionnementsexuel. En ce sens, l'objectif premier de la présente étude est d'identifier les différents profils de répercussions sexuelles chez des femmes victimes d'ASA. L'objectif secondaire est de vérifier si les variables sociodémographiques et la nature de l'attachement amoureux sont associées à l'appartenance à un profil particulier de séquelles sexuelles. 


\section{Méthode}

Recrutées à l'Université Laval et à l'Université de Montréal, 150 femmes d'au moins 18 ans ayant vécu une ASA ont rempli, sur une période maximale de trois semaines, des questionnaires en ligne (voir Tableau 1). Les variables d'intérêt étaient certaines variables sociodémographiques, la détresse sexuelle, la compulsion sexuelle, l'évitement sexuel, les perturbations sexuelles, la satisfaction sexuelle, la fonction sexuelle ainsi que les deux dimensions de l'attachement.

\section{Résultats et discussion}

Des analyses de profils latents ont été effectuées à l'aide du logiciel Mplus version 8.0 afin de déterminer le nombre de profils représentant le mieux l'échantillon. L'examen des indices d'ajustement (AIC, BIC, LL, BLRT) et de l'interprétabilité des profils, c'est-à-dire la capacité à distinguer des profils hétérogènes entre eux tout en gardant un maximum d'homogénéité à I'intérieur de ceux-ci, indique que le meilleur modèle est composé de quatre profils latents. Comme illustré à la Figure 1, les profils détresse, compulsion et dysfonction se distinguent selon des types qualitatifs distincts de difficultés sexuelles dominantes. Toutefois, le profil bienêtre regroupe des femmes qui rapportent peu de compulsion sexuelle, d'évitement sexuel, de perturbations sexuelles et de détresse sexuelle ainsi que des niveaux élevés de satisfaction sexuelle et de fonction sexuelle.

Pour ce qui est des variables associées aux profils, les résultats suggèrent qu'il n'existe pas de différence significative entre le statut socioéconomique et l'appartenance à un profil particulier. La majorité des femmes de l'étude étant de bas niveau socioéconomique, le manque de variabilité pourrait expliquer l'absence d'association significative entre les répercussions sexuelles et le statut socioéconomique. Quant au statut relationnel, la proportion de femmes célibataires est significativement plus élevée dans les profils compulsion et dysfonction alors que le profil bien-être regroupe significativement plus de femmes en couple. En ce sens, être en couple pourrait agir comme un facteur de protection pour les femmes à la suite d'une ASA en raison du soutien social que procure la relation. Pour ce qui est de l'attachement, les femmes des profils détresse et compulsion rapportent significativement plus d'anxiété d'abandon que celles du profil bien-être.

\section{Tableau 1}

Instruments de mesure utilisés

\begin{tabular}{|c|c|}
\hline Instruments de mesure & Variables d'intérêt \\
\hline Questionnaire sociodémographique & $\begin{array}{l}\text { Âge, culture, scolarité, occupation principale, } \\
\text { revenu, orientation sexuelle et statut relationnel }\end{array}$ \\
\hline ECR-12 & $\begin{array}{l}\text { Anxiété d'abandon } \\
\text { Évitement de l'intimité }\end{array}$ \\
\hline Échelle de compulsion sexuelle & Compulsion sexuelle \\
\hline $\begin{array}{l}\text { Sous-échelle d'évitement sexuel de l'Échelle } \\
\text { d'aversion sexuelle }\end{array}$ & Évitement sexuel \\
\hline Mesure globale de satisfaction sexuelle & Satisfaction sexuelle \\
\hline $\begin{array}{l}\text { Sous-échelle de perturbations sexuelles } \\
\text { (Inventaire des symptômes associés au trau- } \\
\text { ma-version 2) }\end{array}$ & Perturbations sexuelles \\
\hline $\begin{array}{l}\text { L'Échelle révisée d'évaluation de la détresse } \\
\text { sexuelle chez les femmes }\end{array}$ & Détresse sexuelle \\
\hline Index de la Fonction Sexuelle de la Femme & Fonction sexuelle \\
\hline
\end{tabular}




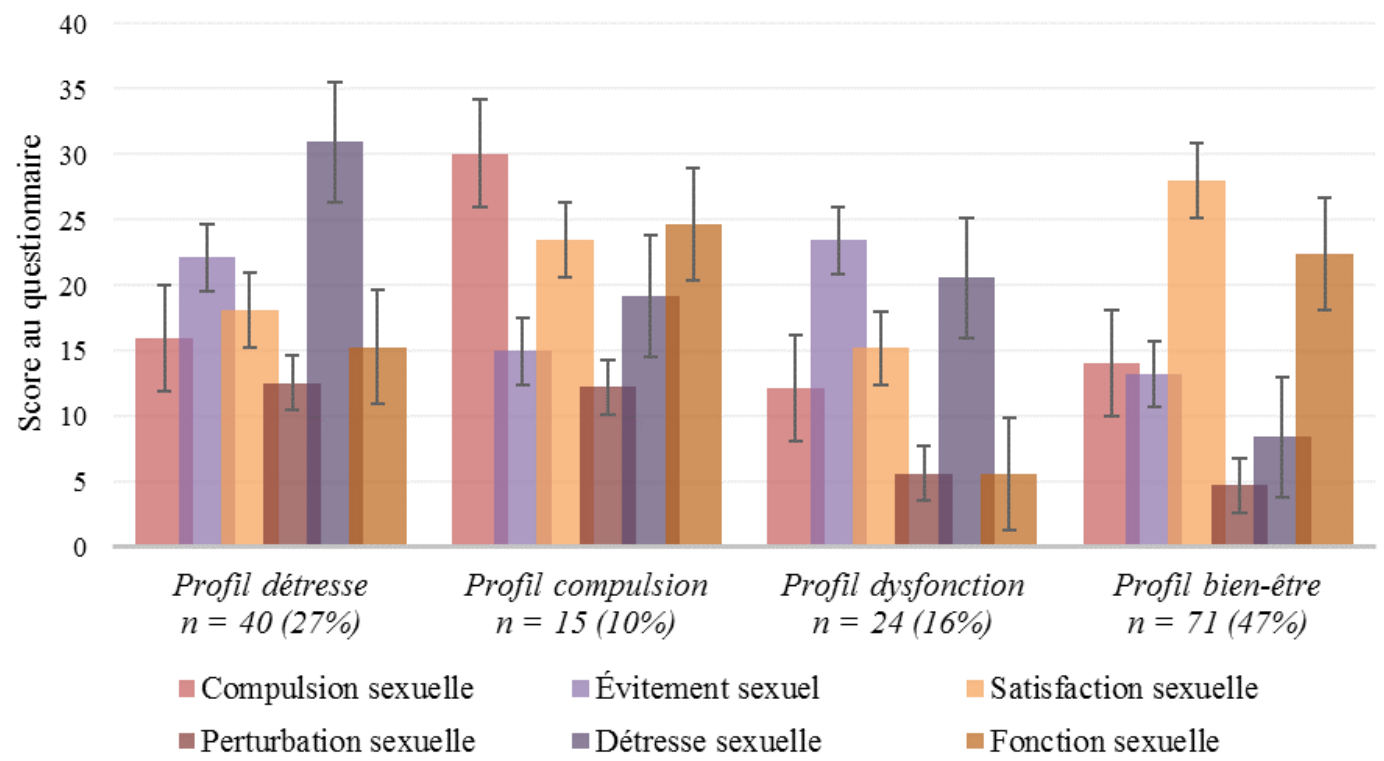

Figure 1. Répercussions sexuelles selon les profils latents. Les barres d'erreur représentent l'erreur standard de la moyenne.

De plus, les femmes du profil compulsion rapportent significativement plus d'anxiété d'abandon que celles du profil dysfonction. Finalement, les résultats suggèrent que les femmes des profils détresse et compulsion ont significativement plus d'évitement de l'intimité que celles du profil bien-être.

Ces résultats appuient que de hauts niveaux d'anxiété d'abandon et d'évitement de l'intimité seraient corrélés à un profil de répercussions sexuelles plus problématique. En effet, le profil bien-être regroupe des femmes présentant significativement moins d'anxiété d'abandon et d'évitement de l'intimité. Celles-ci ont également des répercussions sexuelles moins sévères, ce qui suggère qu'une bonne sécurité d'attachement peut agir comme facteur de protection sur leur sexualité. Puisque le statut relationnel et les dimensions de l'attachement ont une influence sur les répercussions sexuelles vécues par les femmes à la suite d'une ASA, il est essentiel d'en tenir compte pour guider les interventions offertes à ces femmes.

\section{Références}

Brown, N. L., Wilson, S. R., Kao, Y.-M., Luna, V., Kuo, E. S., Rodriguez, C., \& Lavori, P. W. (2003). Correlates of sexual abuse and subsequent risk taking. Hispanic Journal of Behavioral Sciences, 25, 331-351. doi:10.1177/0739986303257147

Burri, A., Schweitzer, R., \& O'Brien, J. (2014). Correlates of female sexual functioning: Adult attachment and differentiation of self. Journal of Sexual Medicine, 11, 2188-2195. doi:10.1111/jsm.12561

Conoscenti, L. M., \& McNally, R. J. (2006). Health complaints in acknowledged and unacknowledged rape victims. Journal of Anxiety Disorders, 20, 372-379. doi:10.1016/ j.janxdis.2005.03.001

Gidycz, C. A., \& Kelley, E. L. (2016). Rape and sexual assault victimization. Dans C. A. Cuevas, \& C. M. Rennison (Eds.), The Wiley-Blackwell Handbook on the Psychology of Violence (pp. 457-481). Hoboken, NJ: John Wiley. doi:10.1002/9781118303092.ch23

Gouvernement du Québec. (2001). Orientations gouvernementales en matière d'agression sexuelle. Récupéré surhttp://www.scf.gouv.qc.ca/ 
fileadmin/publications/Violence/Orientations_gouv_agression_sexuelle_2001.pdf.

Mittal, M., Stockman, J. K., Seplaki, C. L., Thevenet-Morrison, K., Guido, J., \& Carey, M.P. (2013). HIV risk among women from domestic violence agencies: prevalence and correlates. Journal of the Association of Nurses in AIDS Care, 24, 322-330. doi:10.1016/j. jana.2012.11.009

O'Driscoll, C., \& Flanagan, E. (2016). Sexual problems and post-traumatic stress disorder following sexual trauma: A meta-analytic review. Psychology and Psychotherapy: Theory, Research and Practice, 89, 351-367. doi:10.1111/papt.12077
Offman, A., \& Matheson, K. (2004). The sexual self-perceptions of young women experiencing abuse in dating relationships. Sex Roles, 51, 551-560. doi:10.1007/s11199-004 5465-5

Turchik, J. A., \& Hassija, C. M. (2014). Female sexual victimization among college students: assault severity, health risk behaviors, and sexual functioning. Journal of Interpersonal Violence, 29, 2439-2457. doi:10.1177/ 0886260513520230

Wingood, G. M., \& DiClemente, R. J. (1998). Rape among African American women: Sexual, psychological, and social correlates predisposing survivors to risk of STD/HIV. Journal of Women's Health, 7, 77-84. doi:10.1089/ jwh.1998.7.77

\title{
PROFILS LATENTS DE TRAITS DE PERSONNALITÉ CHEZ DES COUPLES EN PSYCHOTHÉRAPIE
}

\author{
Isabelle ARSENEAU1*, Camille ROULEAU ${ }^{1 *}$, Marie-Pierre SAMSON ${ }^{1 *}$, Marie-Ève DASPE ${ }^{2}$ \& \\ Stéphane SABOURIN ${ }^{1}$ \\ 1 École de psychologie, Université Laval, Québec, Canada ${ }^{2}$ Department of Psychology, University of Southern \\ California, Los Angeles, États-Unis \\ *Les trois premières autrices ont fourni une contribution égale à la publication.
}

\section{Introduction}

Modulée tout au long de la vie, la personnalité est responsable des différences individuelles menant à l'adoption de patrons de réponses cognitives, affectives et comportementales (Mischel \& Shoda, 1995). Les dimensions de la personnalité du modèle en cinq facteurs (NEO$\mathrm{FFI}$ ) sont liées à divers aspects du fonctionnement en couple, se répercutant ultimement sur la satisfaction conjugale (Bouchard et coll., 2017).

Tout d'abord, le névrosisme, étant la tendance à vivre des émotions négatives, représente le principal trait nuisant à la satisfaction conjugale, dû à des émotions de tristesse, de colère et d'angoisse (Bouchard et coll., 2017). Ensuite, l'extraversion, constituant la recherche de stimulation sociale, présente à la fois une association positive avec la satisfaction (Malouff, Thorsteinsson, Schutte, Bhullar \& Rooke, 2010) et un lien négatif en raison du risque de socialisation avec de nouvelles personnes (Shiota \& Levenson, 2007). Quant à l'ouverture à l'expérience, soit l'intérêt pour la nouveauté, des liens mitigés ressortent également, associant parfois ce trait au développement de relations extradyadiques plus permissives (Bouchard et coll., 2017), ou encore à une plus grande compréhension vis-à-vis du partenaire (Daspe, Sabourin, Péloquin, Lussier \& Wright, 2013). D'un autre côté, étant liée à des patrons de communication positive, l'amabilité présente un lien positif avec la satisfaction conjugale. Enfin, la propension à être consciencieux, représentant l'aspect motivationnel nécessaire à l'accomplissement d'objectifs, est positivement 